\title{
Null and approximate controllability for weakly blowing up semilinear heat equations
}

by

\author{
Enrique FERNÁNDEZ-CARA ${ }^{\mathrm{a}, 1}$, Enrique ZUAZUA ${ }^{\mathrm{b}, 2}$ \\ a Departamento de Ecuaciones Diferenciales y Análisis Numérico, \\ Universidad de Sevilla, 41080 Sevilla, Spain \\ b Departamento de Matemática Aplicada, Universidad Complutense, \\ 28040 Madrid, Spain
}

Manuscript received 7 October 1999

ABSTRACT. - We consider the semilinear heat equation in a bounded domain of $\mathbb{R}^{d}$, with control on a subdomain and homogeneous Dirichlet boundary conditions. We prove that the system is null-controllable at any time provided a globally defined and bounded trajectory exists and the nonlinear term $f(y)$ is such that $|f(s)|$ grows slower than $|s| \log ^{3 / 2}(1+$ $|s|)$ as $|s| \rightarrow \infty$. For instance, this condition is fulfilled by any function $f$ growing at infinity like $|s| \log ^{p}(1+|s|)$ with $1<p<3 / 2$ (in this case, in the absence of control, blow-up occurs). We also prove that, for some functions $f$ that behave at infinite like $|s| \log ^{p}(1+|s|)$ with $p>2$, null controllability does not hold. The problem remains open when $f$ behaves at infinity like $|s| \log ^{p}(1+|s|)$, with $3 / 2 \leqslant p \leqslant 2$. Results of the same

\footnotetext{
${ }^{1}$ E-mail: cara@numer.us.es. Supported by grants PB95-1242 and PB98-1134 of the DGES (Spain).

${ }^{2}$ E-mail: zuazua@eucmax.sim.ucm.es. Supported by grant PB96-0663 of the DGES (Spain).
} 
kind are proved in the context of approximate controllability.

(C) 2000 L'Association Publications de l'Institut Henri Poincaré. Published by Elsevier B.V. All rights reserved

AMS classification: 93B05, 93C20

RÉSUMÉ. - On considère l'équation de la chaleur semilinéaire dans un domaine borné de $\mathbb{R}^{d}$, avec un contrôle à support dans un sousdomaine et avec des conditions de Dirichlet au bord. On démontre que, s'il existe une trajectoire bornée et globalement définie et le terme non linéaire $f(y)$ est tel que $|f(s)|$ croît moins vite que $|s| \log ^{3 / 2}(1+|s|)$ quand $|s| \rightarrow \infty$, alors le système est exactement contrôlable à zéro dans un temps arbitrairement petit. Par exemple, cette condition sur $f$ est satisfaite si $f(s)$ croît à l'infini comme $|s| \log ^{p}(1+|s|)$ avec $1<p<3 / 2$ (dans ce cas, en absence de contrôle, on a explosion en temps fini). On démontre aussi que, pour tout $p>2$, on n'a pas la contrôlabilité exacte à zéro pour certaines fonctions $f$ dont le comportement à l'infini est comme celui de $|s| \log ^{p}(1+|s|)$. Cette question reste ouverte lorsque $3 / 2 \leqslant p \leqslant 2$. Finalement, on démontre des résultats du même type dans le contexte de la contrôlabilité approchée.

(C) 2000 L'Association Publications de l'Institut Henri Poincaré. Published by Elsevier B.V. All rights reserved

\section{INTRODUCTION AND MAIN RESULTS}

Let $\Omega \subset \mathbb{R}^{d}$ be a bounded domain with boundary of class $C^{2}$, let $T>0$ be given and assume $f: \mathbb{R} \mapsto \mathbb{R}$ is locally Lipschitz-continuous. We will consider semilinear parabolic systems of the form

$$
\begin{cases}y_{t}-\Delta y+f(y)=v 1_{\omega} & \text { in } \Omega \times(0, T), \\ y=0 & \text { on } \partial \Omega \times(0, T), \\ y(x, 0)=y_{0}(x) & \text { in } \Omega .\end{cases}
$$

In (1.1), $y=y(x, t)$ is the state and $v=v(x, t)$ is a control that acts on the system through the nonempty open set $\omega \subset \Omega .1_{\omega}$ denotes the characteristic function of the set $\omega$. We shall denote by $Q$ the cylinder $\Omega \times(0, T)$ and by $\Sigma$ its lateral boundary $\partial \Omega \times(0, T)$.

We will assume that $y_{0} \in L^{2}(\Omega)$ and $v \in L^{\infty}(\omega \times(0, T))$. In most part of this paper, we will also assume that, for some $y_{0}$ and $v$, system (1.1) admits a solution globally defined in the time interval $[0, T]$. Of course, 
this holds immediately when

$$
f(0)=0,
$$

in which case $y \equiv 0$ solves (1.1), with $y_{0}=0$ and $v \equiv 0$.

In the sequel, $C$ denotes a generic positive constant. For instance, the equality $C=C(\Omega, \omega)$ means that $C$ only depends of $\Omega$ and $\omega$.

For simplicity, we will require $f$ to satisfy

$$
\left|f^{\prime}(s)\right| \leqslant C\left(1+|s|^{p}\right) \quad \text { a.e., with } p \leqslant 1+4 / d .
$$

Under this condition, system (1.1) possesses exactly one local (in time) solution.

In accordance with the results in [5], under the growth condition

$$
|f(s)| \leqslant C(1+|s| \log (1+|s|)) \quad \forall s \in \mathbb{R},
$$

the solutions of (1.1) are globally defined in $[0, T]$. More precisely, one has

$$
y \in C\left([0, T] ; L^{2}(\Omega)\right) \cap L^{2}\left(0, T ; H_{0}^{1}(\Omega)\right) .
$$

This is also true if, instead of (1.4), we assume a "good-sign" condition, like the following:

$$
f(s) s \geqslant-C\left(1+|s|^{2}\right) \quad \forall s \in \mathbb{R} .
$$

The main goal of this paper is to analyze the controllability of (1.1) when blow-up occurs, i.e., without imposing any of these conditions (1.4) or (1.5).

It will be assumed that $\omega \neq \Omega$. Otherwise, when $\omega=\Omega$, the problem can be reduced to the controllability of the (linear) heat equation since the nonlinear term is absorbed by the control in a trivial way.

We will first analyze the so-called null controllability property. System (1.1) is said to be null-controllable at time $T$ if, for any $y_{0} \in L^{2}(\Omega)$ and any globally defined bounded trajectory $y^{*}$ (corresponding to the data $y_{0}^{*} \in L^{2}(\Omega)$ and $\left.v^{*} \in L^{\infty}(\omega \times(0, T))\right)$, there exists a control $v \in$ $L^{\infty}(\omega \times(0, T))$ such that the corresponding solution of (1.1) is also globally defined in $[0, T]$ and satisfies

$$
y(x, T)=y^{*}(x, T) \text { in } \Omega .
$$


Obviously, when (1.1) is linear, this is equivalent to say that, for each $y_{0} \in L^{2}(\Omega)$, there exists $v \in L^{\infty}(\omega \times(0, T))$ such that the corresponding solution $y$ satisfies

$$
y(x, T)=0 \quad \text { in } \Omega .
$$

This justifies the definition we have introduced of null-controllable systems.

Notice that, if (1.6) holds, extending $v$ for $t \geqslant T$ as the control $v^{*}$ associated to $y^{*}$, we obtain a solution $y$ that coincides with $y^{*}$ as long as $y^{*}$ exists. In particular, if $y^{*}$ is a stationary solution of (1.1) or, more generally, $y^{*}$ is defined for all $t \geqslant 0$, then $y$ is also globally defined and coincides with $y^{*}$ for all $t \geqslant T$. Hence, from the viewpoint of applications, it is very important to know whether or not (1.1) is nullcontrollable.

Our first main result is of negative nature.

THEOREM 1.1. - There exist locally Lipschitz-continuous functions $f$ such that $f(0)=0$, satisfying

$$
|f(s)| \sim|s| \log ^{p}(1+|s|) \quad \text { as }|s| \rightarrow \infty
$$

with $p>2$, for which system (1.1) fails to be null-controllable for all $T>0$.

Remark 1.1. - For the proof of Theorem 1.1, we choose $p>2$ and

$$
f(s)=\int_{0}^{|s|} \log ^{p}(1+|\sigma|) \mathrm{d} \sigma \quad \forall s \in \mathbb{R}
$$

and we prove a localized estimate (in $\Omega \backslash \bar{\omega}$ ) that shows that the control cannot compensate the blow-up phenomena occurring in $\Omega \backslash \bar{\omega}$. Arguments of this kind are well known. For instance, see J. Henry [14] for the proof of the lack of approximate controllability of the heat equation with nonlinear absorption terms; see also O.Yu. Imanuvilov [15] and A. Fursikov and O.Yu. Imanuvilov [10] for examples of systems that fail to be null-controllable with power-like nonlinearities, i.e., in the more restrictive class of nonlinear terms growing at infinity like $|s|^{p}$ with $p>1$.

The function $f$ in (1.9) is such that $f(0)=0$. Therefore, $y^{*} \equiv 0$ is a stationary solution of (1.1) corresponding to the control $v^{*} \equiv 0$ and, as mentioned above, the null controllability problem makes sense. 
It is important to observe that the solutions to (1.1) can blow up in the absence of control as soon as $|f(s)|$ grows at infinity as in (1.8) with $p>1$. Therefore, Theorem 1.1 does not exclude the null controllability of a whole range of nonlinear systems for which blow-up occurs. Namely, when (1.8) is satisfied with $1<p \leqslant 2$.

In our second main result, we establish conditions under which (1.1) is null-controllable:

THEOREM 1.2. - Let $T>0$. Assume that (1.1) admits at least one globally defined and bounded solution $y^{*}$, corresponding to the data $y_{0}^{*} \in L^{2}(\Omega)$ and $v^{*} \in L^{\infty}(\omega \times(0, T))$. Assume that $f: \mathbb{R} \mapsto \mathbb{R}$ is locally Lipschitz-continuous and satisfies (1.3) and

$$
\frac{f(s)}{|s| \log ^{3 / 2}(1+|s|)} \rightarrow 0 \quad \text { as }|s| \rightarrow \infty \text {. }
$$

Then (1.1) is null-controllable at time $T$.

Remark 1.2. - There is an extensive literature on semilinear parabolic and elliptic problems analyzing the existence of global and/or stationary solutions. At this respect the following result by $\mathrm{H}$. Brezis et al. [3] is worth mentioning: Assume that, for some $\sigma \geqslant 0$, one has $f(\sigma)<0$ and

$$
\int_{\sigma}^{\infty} \frac{1}{f(s)} \mathrm{d} s>-\infty
$$

Also, assume there exists $y_{0} \in L^{\infty}(\Omega)$, with $y_{0} \geqslant 0$, such that the system (1.1) corresponding to this initial $y_{0}$ and $v \equiv 0$ possesses one global classical solution (defined for all $t \geqslant 0$ ). Then there exists at least one weak stationary solution of (1.1) corresponding to $v \equiv 0$.

Recall that (1.11) guarantees the existence of solutions of (1.1) that blow up in finite time. Roughly speaking, this result shows that, in the class of nonlinearities in which blow-up arises, for $v \equiv 0$, the existence of positive global solutions implies that weak stationary solutions exist. It is a routine argument to show that, under the growth conditions we are imposing on $f$, these weak solutions are bounded.

Remark 1.3. - The proof of Theorem 1.2 provides estimates on the size of the control needed to achieve null controllability. Furthermore, by inspection of the proof, one sees that null controllability still holds under slightly more general conditions. More precisely, for each globally 
defined and bounded solution $y^{*}$, there exists $\ell\left(y^{*}\right)>0$ such that, if

$$
\limsup _{|s| \rightarrow \infty} \frac{|f(s)|}{|s| \log ^{3 / 2}(1+|s|)} \leqslant \ell\left(y^{*}\right)<+\infty
$$

and $y_{0}$ is given, we can find $v^{*} \in L^{\infty}(\omega \times(0, T))$ such that (1.6) is satisfied.

Remark 1.4. - In particular, Theorem 1.2 says that, for each $y_{0} \in$ $L^{2}(\Omega)$, there exists a control $v$ such that the corresponding solution of (1.1) is globally defined in $[0, T]$. In other words, if a globally defined trajectory exists, then for each $y_{0}$ a control $v$ can be found such that the corresponding solution is globally defined as well. Of course, this cannot be guaranteed to hold for any right-hand side and any initial datum, since we are in the range in which blow-up may occur.

Remark 1.5. - Without the assumption (1.3) on $f$, the uniqueness of a solution to (1.1) for a given $v$ is not guaranteed. In these conditions, arguing as in the proof of Theorem 1.2, we can deduce the existence of a control $v$ such that system (1.1) admits at least one solution that is globally defined in $[0, T]$ and satisfies (1.6). We refer to [16] for a similar discussion.

Theorem 1.2 asserts that system (1.1) is null-controllable under the growth condition (1.8) provided $p<3 / 2$. Obviously, there is a gap between Theorems 1.1 and 1.2: We do not know whether or not system (1.1) is null-controllable when $f$ satisfies (1.8) with $3 / 2 \leqslant p \leqslant 2$.

As we shall see, the proof we give of Theorem 1.1 does not hold for $p \leqslant 2$. The same can be said about the proof of Theorem 1.2 when $p \geqslant 3 / 2$. Thus, the case $3 / 2 \leqslant p \leqslant 2$ is an interesting open problem. We shall return to it in Section 6.

In [8], it was shown that zero controllability holds under the more restrictive condition

$$
\frac{f(s)}{|s| \log (1+|s|)} \rightarrow 0 \quad \text { as }|s| \rightarrow \infty .
$$

Note that, under condition (1.12), the solutions to (1.1) cannot blow up. Recently, V. Barbu [2] has proved the zero controllability of (1.1) under the growth condition (1.10), but imposing additional "good-sign" conditions similar to (1.5) (see also [1]). Therefore, to our knowledge, Theorem 1.2 is the first result in the literature on the null controllability of blowing-up semilinear heat equations. 
Recall that, in the context of the semilinear wave equation, due to the finite speed propagation property, if blow-up occurs, exact controllability cannot hold (see [19]). Thus, Theorem 1.2 holds due to the parabolic nature of the equation under consideration.

For the proof of Theorem 1.2, we will use the explicit estimates on the cost of controllability obtained in [9] and the fixed point method introduced in the context of the semilinear wave equation in [17] and later applied to semilinear heat equations in [7,8] and [18] (see also [10]). Recall that the estimates in [9] were obtained by adapting the global Carleman inequalities in [10].

However, when applying the fixed point argument, we introduce a new ingredient to avoid blow-up to occur. Indeed, as usual, we first linearize the system and show its controllability analyzing how the control depends of the size of the potential of the linearized equation. Usually, one takes $T$ as the control time for all the linearized equations (see, e.g., [17]). However, in the proof of Theorem 1.2, the control time is chosen depending on the size of the potential so that, roughly speaking, it decreases as this size increases and tends to zero as the size tends to infinity. This is made in order to avoid blow-up phenomena to occur. Note that this strategy is in agreement with common sense: In the presence of blow-up phenomena, one has to act on the system very fast, before blowup occurs.

This idea of taking short control times has been used in [16] for the one-dimensional heat equation with nonlinearities that behave sublinearly at infinity and by O. Glass [13] in the context of the 3-d Euler equations.

Let us now analyze the approximate controllability property. System (1.1) is said to be approximately controllable at time $T$ if, for any $y_{0} \in$ $L^{2}(\Omega), y_{1} \in L^{2}(\Omega)$ and $\varepsilon>0$, there exists a control $v \in L^{\infty}(\omega \times(0, T))$ such that the solution of (1.1) is globally defined in $[0, T]$ and satisfies

$$
\left\|y(\cdot, T)-y_{1}\right\|_{L^{2}(\Omega)} \leqslant \varepsilon
$$

In other words, system (1.1) is approximately controllable if the set of reachable states is dense in $L^{2}(\Omega)$.

In the context of linear heat equations, approximate controllability is a consequence of the null controllability property (see for instance [9]). But this is not necessarily true for semilinear equations.

Our third main result is the following: 
THEOREM 1.3. - There exist locally Lipschitz-continuous functions $f$ satisfying (1.8) with $p>2$, such that, whatever $T>0$ is, system (1.1) is not approximately controllable at time $T$.

Remark 1.6. - The proof of Theorem 1.3 is similar to the proof of Theorem 1.1. In this case, we introduce the following function $f$ :

$$
f(s)=\int_{0}^{s} \log ^{p}(1+|\sigma|) \mathrm{d} \sigma \quad \forall s \in \mathbb{R} .
$$

The absorption effect of the nonlinearity allows to prove that, for any $y_{0} \in L^{2}(\Omega)$, the set of reachable states are uniformly bounded away from the control subdomain $\omega$. Recall that, when $f(s)=|s|^{r-1} s$ for some $r>1$, this was already observed by A. Bamberger (see for instance [14]).

Remark 1.7. - It would also be interesting to know whether blowup phenomena can be an obstruction to approximate controllability even when the initial data are small. In particular, let us assume that $y_{0}=0$ and let $f$ be as in (1.9) with $p>2$. We do not know if the following is true: For any $y_{1} \in L^{2}(\Omega)$ and any $\varepsilon>0$, there exists a control $v \in$ $L^{\infty}(\omega \times(0, T))$ such that the corresponding solution of $(1.1)$ is globally defined in $[0, T]$ and satisfies (1.13).

We also have the following result:

THEOREM 1.4. - Let $T>0$ and let the assumptions of Theorem 1.2 be satisfied. Then (1.1) is approximately controllable at time $T$.

Let us briefly describe the strategy of proof of Theorem 1.4. We will argue as follows. Let $y_{0}, y_{1}$ and $\varepsilon>0$ be given and assume that $y_{1}$ is regular enough. We divide the time interval $[0, T]$ in two parts. In the first (large) subinterval $[0, T-\delta]$, we choose $v_{1}$ such that $y$ satisfies (1.6), with $y^{*}$ being the globally defined solution whose existence is assumed. In the second one, $[T-\delta, T]$, we apply a control $v_{2}$ that drives the solution from $y^{*}(\cdot, T-\delta)$ to $w(\cdot, T)$ exactly. Here, $w$ is the solution of the auxiliary problem

$$
\begin{cases}w_{t}-\Delta w+f(w)=0 & \text { in } \Omega \times(T-\delta, T), \\ w=0 & \text { on } \partial \Omega \times(T-\delta, T), \\ w(x, T-\delta)=y_{1}(x) & \text { in } \Omega\end{cases}
$$


The existence of $v_{1}$ and $v_{2}$ is implied by Theorem 1.2. Furthermore, if $\delta$ is sufficiently small, we have

$$
\left\|w(\cdot, T)-y_{1}\right\|_{L^{2}(\Omega)} \leqslant \varepsilon .
$$

Consequently, by setting $v=v_{1}$ for $t \in(0, T-\delta)$ and $v=v_{2}$ for $t \in$ $(T-\delta, T)$ we will have found a control $v$ such that the corresponding solution to (1.1) satisfies (1.13).

Analyzing this proof, we see that the main ingredients needed for the proof to work are (a) there exists a trajectory starting from $y_{0}$ defined in the whole time interval $[0, T-\delta]$ and (b) the system is null-controllable in any arbitrarily short time interval. Since (1.4) avoids the presence of any blow-up phenomena, we find that an immediate consequence of Theorem 1.4 is the following:

COROLlary 1.1. - Let $T>0$. Assume that $f$ is locally Lipschitzcontinuous and satisfies (1.3) and (1.4). Then (1.1) is approximately controllable at time $T$.

The rest of this paper is organized as follows. Section 2 is devoted to prove Theorem 1.1. In Section 3, we prove Theorem 1.2. In Section 4, we prove Theorem 1.3. Section 5 is concerned with the proof of Theorem 1.4. Finally, in Section 6, we discuss in detail the open problem mentioned above $(f$ satisfies $(1.8)$ with $3 / 2 \leqslant p \leqslant 2)$ and other related issues.

\section{PROOF OF THE LACK OF NULL CONTROLLABILITY}

This section is devoted to prove Theorem 1.1. Let us introduce the following function $f$ :

$$
f(s)=\int_{0}^{|s|} \log ^{p}(1+\sigma) \mathrm{d} \sigma \quad \forall s \in \mathbb{R},
$$

with

$$
p>2 .
$$

Obviously, $f$ is convex and $f(s) s<0$ for $s<0$. On the other hand,

$$
f(s) \sim|s| \log ^{p}(1+|s|) \quad \text { as }|s| \rightarrow \infty .
$$


Therefore, $f$ is in the range of nonlinearities in which blow-up occurs in the absence of control, i.e., with $v \equiv 0$. The proof of Theorem 1.1 is based on the fact that there are initial data which lead to blow-up before time $T$, whatever the control $v$ is.

We proceed as in [10], Section I.5 (see also [14] for a similar argument in the context of approximate controllability). Thus, we introduce a nonnegative function $\rho \in \mathcal{D}(\Omega)$ such that

$$
\rho=0 \quad \text { in } \omega, \quad \int_{\Omega} \rho \mathrm{d} x=1 .
$$

Let $v \in L^{\infty}(\omega \times(0, T))$ be given and let $y$ be the solution to (1.1). Let us multiply by $\rho$ the equation satisfied by $y$ and let us integrate over $\Omega$. Taking into account that the control is supported by $\omega \times(0, T)$, we obtain:

$$
\frac{\mathrm{d}}{\mathrm{d} t} \int_{\Omega} \rho y \mathrm{~d} x=\int_{\Omega} \rho(\Delta y) \mathrm{d} x-\int_{\Omega} \rho f(y) \mathrm{d} x .
$$

Moreover,

$$
\int_{\Omega} \rho(\Delta y) \mathrm{d} x=\int_{\Omega}(\Delta \rho) y \mathrm{~d} x .
$$

From (2.3), (2.4) and the definition of $f$, we have

$$
\frac{\mathrm{d}}{\mathrm{d} t}\left(-\int_{\Omega} \rho y \mathrm{~d} x\right)=-\int_{\Omega}(\Delta \rho) y \mathrm{~d} x+\int_{\Omega} \rho f(|y|) \mathrm{d} x .
$$

Taking into account that $f$ is convex, we can introduce its convex conjugate $f^{*}$. For the moment, let us assume that

$$
\rho f^{*}(2 \Delta \rho / \rho) \in L^{1}(\Omega)
$$

(we shall return to (2.6) later on). Then, from Young's inequality, we have

$$
\begin{aligned}
\left|\int_{\Omega}(\Delta \rho) y \mathrm{~d} x\right| & \leqslant \int_{\Omega} \rho\left|\frac{\Delta \rho}{\rho}\right||y| \mathrm{d} x \\
& \leqslant \frac{1}{2} \int_{\Omega} \rho f^{*}(2|\Delta \rho| / \rho) \mathrm{d} x+\frac{1}{2} \int_{\Omega} \rho f(|y|) \mathrm{d} x .
\end{aligned}
$$


Let us set

$$
k:=\frac{1}{2} \int_{\Omega} \rho f^{*}(2|\Delta \rho| / \rho) \mathrm{d} x,
$$

which is finite according to (2.6). From (2.5), (2.7) and (2.8), the following is deduced:

$$
\begin{aligned}
\frac{\mathrm{d}}{\mathrm{d} t}\left[-\int_{\Omega} \rho y \mathrm{~d} x\right] & \geqslant-\left|\int_{\Omega}(\Delta \rho) y \mathrm{~d} x\right|+\int_{\Omega} \rho f(|y|) \mathrm{d} x \\
& \geqslant-k+\frac{1}{2} \int_{\Omega} \rho f(|y|) \mathrm{d} x .
\end{aligned}
$$

From Jensen's inequality, we also have

$$
\int_{\Omega} \rho f(|y|) \mathrm{d} x \geqslant f\left(\int_{\Omega} \rho|y| \mathrm{d} x\right)
$$

On the other hand, taking into account that $f$ is increasing on $[0, \infty)$, we know that

$$
f\left(\int_{\Omega} \rho|y| \mathrm{d} x\right) \geqslant f\left(\left|\int_{\Omega} \rho y \mathrm{~d} x\right|\right)=f\left(-\int_{\Omega} \rho y \mathrm{~d} x\right) .
$$

Thus, if we set $z(t)=-\int_{\Omega} \rho(x) y(x, t) \mathrm{d} x$ for all $t$ and $z_{0}=-\int_{\Omega} \rho(x) \times$ $y_{0}(x) \mathrm{d} x$, we find that

$$
\left\{\begin{array}{l}
z^{\prime}(t) \geqslant-k+\frac{1}{2} f(z(t)) \\
z(0)=z_{0}
\end{array}\right.
$$

We are now going to use (2.9) to prove that, for appropriate initial data, $z$ blows up at a finite time. More precisely, let $y_{0} \in L^{2}(\Omega)$ be such that

$$
z_{0}=-\int_{\Omega} \rho(x) y_{0}(x) \mathrm{d} x>0, \quad f\left(z_{0}\right)>2 k
$$

and assume that $z:\left[0, T_{*}\right) \mapsto \mathbb{R}$ is a $C^{1}$ function satisfying (2.9). Let us see that $T_{*}<+\infty$. 
594 E. FERNÁNDEZ-CARA, E. ZUAZUA / Ann. Inst. Henri Poincaré 17 (2000) 583-616

The function $z$ is nondecreasing. Furthermore, if we set

$$
G\left(z_{0} ; s\right):=\int_{z_{0}}^{s} \frac{2}{f(\sigma)-2 k} \mathrm{~d} \sigma \quad \forall s \geqslant z_{0},
$$

then we find that

$$
\frac{\mathrm{d}}{\mathrm{d} t} G\left(z_{0} ; z(t)\right)=\frac{2 z^{\prime}(t)}{f(z(t))-2 k} \geqslant 1 \quad \forall t \in\left[0, T_{*}\right) .
$$

Notice that, in view of (2.1),

$$
f(\sigma) \sim \sigma \log ^{p}(1+\sigma) \quad \text { as } \sigma \rightarrow+\infty, \text { with } p>2 .
$$

Consequently,

$$
G\left(z_{0} ;+\infty\right)=\int_{z_{0}}^{+\infty} \frac{2}{f(\sigma)-2 k} \mathrm{~d} \sigma<+\infty .
$$

Going back to (2.10), we see that

$$
G\left(z_{0} ; z(t)\right)-G\left(z_{0} ; z_{0}\right)=G\left(z_{0} ; z(t)\right) \geqslant t \quad \forall t \in\left[0, T_{*}\right) .
$$

Combining (2.11) and (2.12), we deduce that $z$ blows up in finite time and, therefore, $y$ blows up in $L^{1}(\Omega)$.

In fact, we have found the following estimate for the maximal time of existence:

$$
T_{*} \leqslant \int_{z_{0}}^{+\infty} \frac{2}{f(\sigma)-2 k} \mathrm{~d} \sigma, \quad \text { where } k \text { is given by (2.8). }
$$

Obviously, as $z_{0} \rightarrow \infty$, the blow-up time of $z$ and, consequently, the blow-up time of $y$ in $L^{1}(\Omega)$ tend to zero.

This completes the proof of Theorem 1.1 (assuming that (2.6) is satisfied). Indeed, we have shown that, whatever $T>0$ is, by taking $y_{0} \in L^{2}(\Omega)$ with

$$
z_{0}=-\int_{\Omega} \rho(x) y_{0}(x) \mathrm{d} x \quad \text { sufficiently large, }
$$


the solution $y$ of (1.1) is not globally defined in $[0, T]$, regardless of the choice of the control $v \in L^{\infty}(\omega \times(0, T))$.

It remains to prove that, for the function $f$ in (2.1) (with $p>2$ ), there exists a nonnegative function $\rho \in \mathcal{D}(\Omega)$ satisfying (2.2) and (2.6).

We first claim that

$$
f^{*}(s) \sim p|s|^{1-1 / p} \exp \left(|s|^{1 / p}\right) \quad \text { as } s \rightarrow+\infty .
$$

Indeed, we have by definition that

$$
f^{*}(s)=\sup _{a \in \mathbb{R}}[a s-f(a)]
$$

The supremum in (2.14) is achieved at a critical point $a$ such that $s-f^{\prime}(a)=0$, i.e., $a=\left(f^{\prime}\right)^{-1}(s)$. Thus,

$$
f^{*}(s)=s\left[f^{\prime}\right]^{-1}(s)-f\left(\left[f^{\prime}\right]^{-1}(s)\right) .
$$

According to the definition (2.1), we have

$$
f^{\prime}(r)=\operatorname{sgn}(r) \log ^{p}(1+|r|) \quad \forall r>0 .
$$

Consequently,

$$
f^{*}(s)=s\left[\exp \left(s^{1 / p}\right)-1\right]-\int_{0}^{\exp \left(s^{1 / p}\right)-1} \log ^{p}(1+\sigma) \mathrm{d} \sigma
$$

and it is easy to deduce (2.13) from (2.15) applying l'Hopital's rule.

Let us prove the existence of the desired function $\rho$ taking into account the asymptotic shape of $f^{*}$.

We will first discuss the one-dimensional case $(d=1)$. Obviously, to show that (2.6) holds, the unique delicate point concerns the behavior of $f^{*}\left(2\left|\rho^{\prime \prime}\right| / \rho\right)$ when $\rho$ vanishes. Note that we can always choose $\rho$ supported by an interval $I \subset \Omega \backslash \omega$ and strictly positive in the interior of $I$. Thus, the difficulties arise only at the extremes of the interval $I$. Without loss of generality, we may assume that we are considering the lower extreme, located at $x=0$. We claim that it is then sufficient to take $\rho$ behaving like $\exp \left(-x^{-m}\right)$ with $m>2 /(p-2)$ (recall that $p>2$ ). Indeed, if

$$
\rho(x)=\exp \left(-x^{-m}\right)
$$


then

$$
\rho^{\prime}(x)=m x^{-(m+1)} \exp \left(-x^{-m}\right),
$$

and

$$
\rho^{\prime \prime}(x)=\left[-m(m+1) x^{-(m+2)}+m^{2} x^{-(2 m+2)}\right] \exp \left(-x^{-m}\right) .
$$

Consequently

$$
\begin{aligned}
\frac{\left|\rho^{\prime \prime}(x)\right|}{\rho(x)} & =\left|m^{2} x^{-(2 m+2)}-m(m+1) x^{-(m+2)}\right| \\
& \sim m^{2} x^{-(2 m+2)} \quad \text { as } x \rightarrow 0^{+} .
\end{aligned}
$$

Then, according to (2.13) and (2.16):

$$
\begin{aligned}
f^{*} & \left(2\left|\rho^{\prime \prime}(x)\right| / \rho(x)\right) \\
& \sim p m^{2(1-1 / p)} x^{-(2 m+2)(p-1) / p} \exp \left(m^{2 / p} x^{-(2 m+2) / p}\right) \text { as } x \rightarrow 0^{+} .
\end{aligned}
$$

From (2.17), we see that $\rho f^{*}\left(2\left|\rho^{\prime \prime}(x)\right| / \rho\right) \in L^{1}(\Omega)$ if and only if $m>$ $(2 m+2) / p$ or, equivalently, $m>2 /(p-2)$, as we have chosen above. This proves our assertion.

In several space dimensions, without loss of generality, we may assume that the ball $B(0 ; r)$ is contained in $\Omega \backslash \bar{\omega}$. Then, we can choose $\rho$ behaving like $\exp \left(-(r-|x|)^{-m}\right)$ as $|x| \rightarrow r^{-}$. The same computations above show that (2.6) is satisfied when $m>2 /(p-2)$.

\section{PROOF OF THE NULL CONTROLLABILITY RESULT}

This section is devoted to prove Theorem 1.2. First of all, we will recall some observability estimates obtained in [9] as a consequence of appropriate global Carleman inequalities. Then, we will deduce some refined versions of these observability estimates. This will serve to prove null controllability results (and estimates) for linear heat equations with bounded potentials, with controls in $L^{\infty}(\omega \times(0, T))$. Finally, we will apply a fixed point argument and we will deduce the desired null controllability result for the semilinear heat equation.

\subsection{Preliminaries on observability inequalities}

Let us consider the adjoint system

$$
\begin{cases}-\varphi_{t}-\Delta \varphi+a \varphi=0 & \text { in } Q, \\ \varphi=0 & \text { on } \Sigma, \\ \varphi(T)=\varphi^{0} & \text { in } \Omega .\end{cases}
$$


In (3.1), $a=a(x, t)$ is a potential. We assume $a \in L^{\infty}(Q)$ and $\varphi^{0} \in$ $L^{2}(\Omega)$. The following result was proved in [9]:

Proposition 3.1. - There exists $C=C(\Omega, \omega)>0$ such that

$$
\begin{aligned}
& \|\varphi(\cdot, 0)\|_{L^{2}(\Omega)}^{2} \\
& \quad \leqslant \exp \left[C\left(1+\frac{1}{T}+T\|a\|_{\infty}+\|a\|_{\infty}^{2 / 3}\right)\right] \iint_{\omega \times(0, T)}|\varphi|^{2} \mathrm{~d} x \mathrm{~d} t
\end{aligned}
$$

for any $\varphi^{0} \in L^{2}(\Omega)$ and $T>0$, with $\varphi$ being the solution of (3.1).

The proof of this result requires appropriate global Carleman inequalities, as in [10]. It is important to observe that (3.2) provides precise estimates on how the observability constant depends on $T$ and the size of the potential $a$. This will be essential when dealing with the semilinear problem (1.1) and in particular, when dealing with nonlinearities that may lead to blow-up phenomena.

However, in order to prove Theorem 1.2, we need a refined version of the observability inequality (3.2). This will be obtained in the next section.

\subsection{A refined observability inequality}

The following holds:

Proposition 3.2. - There exists $C=C(\Omega, \omega)>0$ such that

$$
\begin{aligned}
\|\varphi(\cdot, 0)\|_{L^{2}(\Omega)}^{2} \leqslant & \exp \left[C\left(1+\frac{1}{T}+T+\left(T^{1 / 2}+T\right)\|a\|_{\infty}+\|a\|_{\infty}^{2 / 3}\right)\right] \\
& \times\left(\iint_{\omega \times(0, T)}|\varphi| \mathrm{d} x \mathrm{~d} t\right)^{2}
\end{aligned}
$$

for any $\varphi^{0} \in L^{2}(\Omega)$ and $T>0$.

Proof. - We will proceed in several steps.

Step 1. - Let $\omega^{\prime}$ be a nonempty open set satisfying $\omega^{\prime} \Subset \omega$.

Notice that, as an immediate consequence of (3.2), the following observability inequality holds with, possibly, a larger constant $C$ :

$$
\begin{aligned}
& \|\varphi(\cdot, 0)\|_{L^{2}(\Omega)}^{2} \\
& \quad \leqslant \exp \left[C\left(1+\frac{1}{T}+T\|a\|_{\infty}+\|a\|_{\infty}^{2 / 3}\right)\right] \iiint_{\omega^{\prime} \times\left(\frac{T}{3}, \frac{2 T}{3}\right)}|\varphi|^{2} \mathrm{~d} x \mathrm{~d} t .
\end{aligned}
$$


Indeed, from Proposition 3.1 applied to $\omega^{\prime}$ in the time interval $\left[\frac{T}{3}, \frac{2 T}{3}\right]$, we deduce that

$$
\begin{aligned}
& \|\varphi(\cdot, T / 3)\|_{L^{2}(\Omega)}^{2} \leqslant \\
& \quad \exp \left[C\left(1+\frac{3}{T}+\frac{T}{3}\|a\|_{\infty}+\|a\|_{\infty}^{2 / 3}\right)\right] \iint_{\omega^{\prime} \times\left(\frac{T}{3}, \frac{2 T}{3}\right)}|\varphi|^{2} \mathrm{~d} x \mathrm{~d} t .
\end{aligned}
$$

On the other hand, classical $L^{2}$ estimates imply the following for any solution of (3.1):

$$
\|\varphi(\cdot, 0)\|_{L^{2}(\Omega)}^{2} \leqslant \exp \left(\frac{2 T}{3}\|a\|_{\infty}\right)\|\varphi(\cdot, T / 3)\|_{L^{2}(\Omega)}^{2} .
$$

Combining (3.5) and (3.6), we see that (3.4) holds.

Step 2. - Let us prove that

$$
\begin{aligned}
& \iint_{\omega^{\prime} \times\left(\frac{T}{3}, \frac{2 T}{3}\right)}|\varphi|^{2} \mathrm{~d} x \mathrm{~d} t \\
& \leqslant C T^{\alpha}\left(1+T^{1 / 2}\left(1+\|a\|_{\infty}\right)\right)^{\beta}\left(\iint_{\omega \times(0, T)}|\varphi| \mathrm{d} x \mathrm{~d} t\right)^{2}
\end{aligned}
$$

for any $\varphi^{0} \in L^{2}(\Omega)$, where $\alpha$ and $\beta$ are positive numbers only depending of $d$.

To this end, we first claim that, whenever the nonempty open sets $\omega_{i}$, the parameters $\delta_{i}$ and the exponents $r_{i}$ satisfy

$$
\omega^{\prime} \subset \omega_{0} \Subset \omega_{1} \subset \omega, \quad 0<\delta_{1}<\delta_{0}<\frac{T}{2}, \quad 1 \leqslant r_{1}<r_{0}<\infty
$$

and

$$
\left(\frac{d}{2}+1\right)\left[\frac{1}{r_{1}}-\frac{1}{r_{0}}\right]<\frac{1}{2}
$$

we have

$$
\begin{aligned}
& \left(\underset{\omega_{0} \times\left(\delta_{0}, T-\delta_{0}\right)}{ }|\varphi|^{r_{0}} \mathrm{~d} x \mathrm{~d} t\right)^{1 / r_{0}} \\
& \leqslant C T^{\gamma}\left(1+T^{1 / 2}\left(1+\|a\|_{\infty}\right)\right)\left(\int_{\omega_{1} \times\left(\delta_{1}, T-\delta_{1}\right)}|\varphi|^{r_{1}} \mathrm{~d} x \mathrm{~d} t\right)^{1 / r_{1}}
\end{aligned}
$$


for all $\varphi^{0} \in L^{2}(\Omega)$, where $C=C\left(\Omega, \omega_{i}, \delta_{i}, r_{i}, d\right)$ and $\gamma=\gamma\left(r_{i}, d\right), i=$ 0,1 .

Indeed, let us introduce a function $\theta \in \mathcal{D}\left(\omega_{1} \times\left(\delta_{1}, T-\delta_{1}\right)\right)$ such that $\theta \equiv 1$ in $\omega_{0} \times\left(\delta_{0}, T-\delta_{0}\right)$ and $0 \leqslant \theta \leqslant 1$ everywhere. Let us set $\psi=\theta \varphi$. Then

$$
\begin{cases}\psi_{t}+\Delta \psi=a \psi+\left[\theta_{t}+\Delta \theta\right] \varphi+2 \nabla \theta \cdot \nabla \varphi & \text { in } Q, \\ \psi=0 & \text { on } \Sigma, \\ \psi(x, T)=0 & \text { in } \Omega .\end{cases}
$$

In order to simplify the computations, let us reverse the sense of time. More precisely, let us put $\widetilde{\psi}(x, t)=\psi(x, T-t)$. We also introduce $\widetilde{\varphi}$ and $\widetilde{\theta}$, defined in a similar way. We then have

$$
\begin{cases}\widetilde{\psi}_{t}-\Delta \widetilde{\psi}=-a \widetilde{\psi}+\left[\widetilde{\theta}_{t}-\Delta \widetilde{\theta}\right] \widetilde{\varphi}-2 \nabla \widetilde{\theta} \cdot \nabla \widetilde{\varphi} & \text { in } Q, \\ \widetilde{\psi}=0 & \text { on } \Sigma, \\ \widetilde{\psi}(x, 0)=0 & \text { in } \Omega .\end{cases}
$$

Let us denote by $\{S(t)\}_{t \geqslant 0}$ the semigroup generated by the heat equation with Dirichlet boundary conditions. Then

$$
\begin{aligned}
\widetilde{\psi}(\cdot, t)= & \int_{0}^{t} S(t-s)\left\{-a \widetilde{\psi}+\left[\widetilde{\theta}_{t}(s)-\Delta \widetilde{\theta}(s)\right] \widetilde{\varphi}(s)\right. \\
& -2 \nabla \widetilde{\theta} \cdot \nabla \widetilde{\varphi}\}(\cdot, s) \mathrm{d} s
\end{aligned}
$$

Taking $L^{r_{0}}$-norms in (3.10) and using the $L^{r_{1}}-L^{r_{0}}$ regularizing effect of the heat equation, we obtain the following for all $t \in\left(\delta_{1}, T-\delta_{1}\right)$ :

$$
\begin{aligned}
& \|\widetilde{\psi}(\cdot, t)\|_{L^{r_{0}(\Omega)}} \\
& \leqslant C\left[\|a\|_{\infty} \int_{0}^{t}(t-s)^{-\frac{d}{2}\left(\frac{1}{r_{1}}-\frac{1}{r_{0}}\right)}\|\widetilde{\psi}(\cdot, s)\|_{L^{r_{1}(\Omega)}} \mathrm{d} s\right. \\
& \left.\quad+\int_{\delta_{1}}^{t}\left[(t-s)^{-\frac{d}{2}\left(\frac{1}{r_{1}}-\frac{1}{r_{0}}\right)}+(t-s)^{-\frac{d}{2}\left(\frac{1}{r_{1}}-\frac{1}{r_{0}}\right)-\frac{1}{2}}\right]\|\widetilde{\varphi}(\cdot, s)\|_{L^{r_{1}(\omega)}} \mathrm{d} s\right] .
\end{aligned}
$$

The constant $C$ in (3.11) depends on the $L^{\infty}$-norm of $\widetilde{\theta}_{t}, \Delta \widetilde{\theta}$ and $\nabla \widetilde{\theta}$. Therefore, it is determined by $\omega_{i}$ and $\delta_{i}, i=0,1$. This gives

$$
\|\widetilde{\psi}(\cdot, t)\|_{L^{r_{0}(\Omega)}} \leqslant C T^{-1}\left[1+T^{1 / 2}+T^{1 / 2}\|a\|_{\infty}\right]
$$




$$
\times \int_{\delta_{1}}^{t}(t-s)^{-\frac{d}{2}\left(\frac{1}{r_{1}}-\frac{1}{r_{0}}\right)-\frac{1}{2}}\|\widetilde{\varphi}(\cdot, s)\|_{L^{r_{1}(\omega)}} \mathrm{d} s
$$

for all $t \in\left(\delta_{1}, T-\delta_{1}\right)$. Now, from Young's inequality, we find that

$$
\begin{aligned}
& \|\widetilde{\psi}\|_{L^{r_{0}\left(\Omega \times\left(\delta_{1}, T-\delta_{1}\right)\right)}} \\
& \quad \leqslant C T^{-\alpha}\left(1+T^{1 / 2}\left(1+\|a\|_{\infty}\right)\right)\|\varphi\|_{L^{r_{1}\left(\omega_{1} \times\left(\delta_{1}, T-\delta_{1}\right)\right)},}
\end{aligned}
$$

where $\alpha=\left(\frac{d}{2}+1\right)\left(\frac{1}{r_{1}}-\frac{1}{r_{0}}\right)+\frac{1}{2}$. This is possible because

$$
\frac{d}{2}\left(\frac{1}{r_{1}}-\frac{1}{r_{0}}\right)+\frac{1}{2}+\frac{1}{r_{1}}<1+\frac{1}{r_{0}},
$$

which is equivalent to (3.8). The desired estimate (3.9) follows immediately from (3.12).

We cannot apply (3.9) directly to deduce (3.7), since the exponents $r_{1}=1$ and $r_{0}=2$, may not satisfy condition (3.8). Thus, we will apply (3.9) recurrently.

Let us set $r_{0}=2$ and let $r_{1}, r_{2}, \ldots$ be given by

$$
\frac{1}{r_{i}}=\frac{i}{2(d+2)}+\frac{1}{2} \text { for all } i
$$

For an appropriate $I \geqslant 0$, one has $r_{I}>1$ and $r_{I+1} \leqslant 1$. Let us redefine $r_{I+1}$ by setting $r_{I+1}=1$. Let us introduce $\delta>0$ so that $[T / 3-I \delta, 2 T / 3+$ $I \delta] \subset[0, T]$ and also a finite family of increasing open sets $\omega_{i}$, with

$$
\omega^{\prime}=\omega_{0} \Subset \omega_{1} \Subset \cdots \Subset \omega_{I+1}=\omega .
$$

For each $i=0,1, \ldots, I-1$, we can use (2.9) with $\omega_{0}, \omega_{1}, \delta_{0}, \delta_{1}, r_{0}$ and $r_{1}$ respectively replaced by $\omega_{i}, \omega_{i+1}, i \delta,(i+1) \delta, r_{i}$ and $r_{i+1}$. In this way, it is immediate to deduce the inequality (3.7) with $\beta=I$ and $\alpha$ being the sum of the exponents $\gamma$ arising in (3.9) at each step.

Step 3. - The inequalities (3.4) and (3.7) give together

$$
\|\varphi(\cdot, 0)\|_{L^{2}(\Omega)}^{2} \leqslant K\left(\Omega, \omega, d, T,\|a\|_{\infty}\right)\left(\iint_{\omega \times(0, T)}|\varphi| \mathrm{d} x \mathrm{~d} t\right)^{2},
$$

where 
E. FERNÁNDEZ-CARA, E. ZUAZUA / Ann. Inst. Henri Poincaré 17 (2000) 583-616 601

$$
\begin{aligned}
K\left(\Omega, \omega, d, T,\|a\|_{\infty}\right)= & \exp \left[C\left(1+\frac{1}{T}+T\|a\|_{\infty}+\|a\|_{\infty}^{2 / 3}\right)\right] \\
& \times T^{\alpha}\left(1+T^{1 / 2}\left(1+\|a\|_{\infty}\right)\right)^{\beta} .
\end{aligned}
$$

This implies immediately that (3.3) holds for all $\varphi^{0} \in L^{2}(\Omega)$. This completes the proof of Proposition 3.2.

\subsection{Null controllability of the linear heat equation with a potential}

For the proof of Theorem 1.2, we will use a fixed point argument below. One of the main ingredients is to obtain explicit estimates of the norms of the controls needed to achieve the null controllability of the linear heat equation with a potential.

Let us consider the system:

$$
\begin{cases}y_{t}-\Delta y+a y=v 1_{\omega} & \text { in } Q, \\ y=0 & \text { on } \Sigma, \\ y(x, 0)=y_{0}(x) & \text { in } \Omega,\end{cases}
$$

where $a \in L^{\infty}(Q)$. The following holds:

THEOREM 3.1. - For any $T>0$, any $a \in L^{\infty}(Q)$ and any $y_{0} \in$ $L^{2}(\Omega)$, there exist controls $v \in L^{\infty}(\omega \times(0, T))$ such that the corresponding solution of (3.13) satisfies

$$
y(x, T)=0 \quad \text { in } \Omega .
$$

Furthermore, $v$ can be chosen such that the following estimate holds:

$$
\begin{aligned}
& \|v\|_{L^{\infty}(\omega \times(0, T))} \\
& \quad \leqslant \exp \left[C\left(1+\frac{1}{T}+T+\left(T^{1 / 2}+T\right)\|a\|_{\infty}+\|a\|_{\infty}^{2 / 3}\right)\right]\left\|y_{0}\right\|_{L^{2}(\Omega)} .
\end{aligned}
$$

Proof. - Let us fix $T>0, a \in L^{\infty}(Q)$ and $y_{0} \in L^{2}(\Omega)$. For any $\varepsilon>0$, we consider the following functional:

$$
\begin{aligned}
J_{\varepsilon}\left(\varphi^{0}\right)= & \frac{1}{2}\left[\iint_{\omega \times(0, T)}|\varphi| \mathrm{d} x \mathrm{~d} t\right]^{2}+\varepsilon\left\|\varphi^{0}\right\|_{L^{2}(\Omega)} \\
& +\int_{\Omega} \varphi(x, 0) y_{0}(x) \mathrm{d} x \quad \forall \varphi^{0} \in L^{2}(\Omega) .
\end{aligned}
$$

Here, for each $\varphi^{0} \in L^{2}(\Omega), \varphi$ is the corresponding solution of (3.1). 
It is not difficult to see that $\varphi^{0} \mapsto J_{\varepsilon}\left(\varphi^{0}\right)$ is a continuous and strictly convex function on $L^{2}(\Omega)$. Moreover, $J_{\varepsilon}$ is coercive. In fact, proceeding as in [7], it can be checked that

$$
\liminf _{\left\|\varphi^{0}\right\|_{L^{2}(\Omega)} \rightarrow \infty} \frac{J_{\varepsilon}\left(\varphi^{0}\right)}{\left\|\varphi^{0}\right\|_{L^{2}(\Omega)}} \geqslant \varepsilon .
$$

Therefore, $J_{\varepsilon}$ achieves its minimum at a unique $\widehat{\varphi}_{\varepsilon}^{0} \in L^{2}(\Omega)$. Let $\widehat{\varphi}_{\varepsilon}$ be the associate solution of (3.1). Again arguing as in [7], it is easy to see that, for some

$$
v_{\varepsilon} \in\left(\iint_{\omega \times(0, T)}\left|\widehat{\varphi}_{\varepsilon}\right| \mathrm{d} x \mathrm{~d} t\right) \operatorname{sgn}\left(\widehat{\varphi}_{\varepsilon}\right),
$$

the solution $y_{\varepsilon}$ of (3.13) satisfies

$$
\left\|y_{\varepsilon}(\cdot, T)\right\|_{L^{2}(\Omega)} \leqslant \varepsilon .
$$

We claim that, for a suitable $C=C(\Omega, \omega)>0$, the following holds:

$$
\begin{aligned}
& \left\|v_{\varepsilon}\right\|_{L^{\infty}(\omega \times(0, T))} \\
& \quad \leqslant \exp \left[C\left(1+\frac{1}{T}+T+\left(T^{1 / 2}+T\right)\|a\|_{\infty}+\|a\|_{\infty}^{2 / 3}\right)\right]\left\|y_{0}\right\|_{L^{2}(\Omega)} .
\end{aligned}
$$

Indeed, at the minimum $\widehat{\varphi}_{\varepsilon}^{0}$, we have

$$
J_{\varepsilon}\left(\widehat{\varphi}_{\varepsilon}^{0}\right) \leqslant J_{\varepsilon}(0)=0 .
$$

In accordance with (3.16), we find that

$$
\begin{aligned}
\frac{1}{2}\left[\iint_{\omega \times(0, T)}\left|\widehat{\varphi}_{\varepsilon}\right| \mathrm{d} x \mathrm{~d} t\right]^{2} & \leqslant \int_{\Omega} \widehat{\varphi}_{\varepsilon}(x, 0) y_{0}(x) \mathrm{d} x \\
& \leqslant\left\|\widehat{\varphi}_{\varepsilon}(\cdot, 0)\right\|_{L^{2}(\Omega)}\left\|y_{0}\right\|_{L^{2}(\Omega)}
\end{aligned}
$$

and, therefore,

$$
\begin{aligned}
& \iint_{\omega \times(0, T)}\left|\widehat{\varphi}_{\varepsilon}\right| \mathrm{d} x \mathrm{~d} t \\
& \quad \leqslant \exp \left[C\left(1+\frac{1}{T}+T+\left(T^{1 / 2}+T\right)\|a\|_{\infty}+\|a\|_{\infty}^{2 / 3}\right)\right]\left\|y_{0}\right\|_{L^{2}(\Omega)}
\end{aligned}
$$


(we have used here (3.3)). From (3.19), we obtain (3.18) taking into account that

$$
\left\|v_{\varepsilon}\right\|_{L^{\infty}(\omega \times(0, T))}=\iint_{\omega \times(0, T)}\left|\widehat{\varphi}_{\varepsilon}\right| \mathrm{d} x \mathrm{~d} t .
$$

In view of the uniform bound (3.18), by extracting an appropriate subsequence, we deduce that

$$
v_{\varepsilon} \rightarrow v \quad \text { weakly-* in } L^{\infty}(\omega \times(0, T)),
$$

where $v$ satisfies (3.15). Since we have (3.17) for all $\varepsilon>0$, we deduce that $v$ is such that the solution of (3.13) satisfies (3.14). This ends the proof.

Remark 3.1. - An immediate consequence of Theorem 3.1 is that we can also estimate the minimal norm of a control $v$ needed to drive an initial datum $y_{0}$ to a final state $z(\cdot, T)$, with $z$ being the solution of (3.13) corresponding to an initial datum $z_{0} \in L^{2}(\Omega)$ and a right hand side $w \in L^{\infty}(\omega \times(0, T))$. Indeed, by means of the change of variable $p=y-z$, we see that $v$ drives $y$ from $y_{0}$ to $z(\cdot, T)$ if and only if the control $v=v-w$ drives $p$ from $y_{0}-z_{0}$ to zero. In accordance with Theorem 3.1, we can find such a $v$ satisfying

$$
\begin{aligned}
\|\nu\|_{L^{\infty}(\omega \times(0, T))} \leqslant & \exp \left[C\left(1+\frac{1}{T}+T+\left(T^{1 / 2}+T\right)\|a\|_{\infty}+\|a\|_{\infty}^{2 / 3}\right)\right] \\
& \times\left\|y_{0}-z_{0}\right\|_{L^{2}(\Omega)} .
\end{aligned}
$$

Hence, we have

$$
\begin{aligned}
\|v\|_{L^{\infty}(\omega \times(0, T))} \leqslant & \exp \left[C\left(1+\frac{1}{T}+T+\left(T^{1 / 2}+T\right)\|a\|_{\infty}+\|a\|_{\infty}^{2 / 3}\right)\right] \\
& \times\left\|y_{0}-z_{0}\right\|_{L^{2}(\Omega)}+\|w\|_{L^{\infty}(\omega \times(0, T))} .
\end{aligned}
$$

\subsection{The fixed point method: Conclusion}

Let us complete the proof of Theorem 1.2. Let $y^{*}$ be a bounded and globally defined solution of (1.1), associated to $y_{0}^{*} \in L^{2}(\Omega)$ and $v^{*} \in L^{\infty}(\omega \times(0, T))$. Let us perform the change of variable $p=y-y^{*}$. Then, $y$ solves (1.1) if and only if $p$ satisfies

$$
\begin{cases}p_{t}-\Delta p+f\left(y^{*}+p\right)-f\left(y^{*}\right)=v 1_{\omega} & \text { in } Q, \\ p=0 & \text { on } \Sigma, \\ p(x, 0)=p_{0}(x) & \text { in } \Omega,\end{cases}
$$


where $v=v-v^{*}$ and $p_{0}=y_{0}(x)-y_{0}^{*}$. Our task is then to show that, for each $p_{0} \in L^{2}(\Omega)$, there exists $v \in L^{\infty}(\omega \times(0, T))$ such that

$$
p(x, T)=0 \quad \text { in } \Omega .
$$

We will argue as follows. Let us assume that

$$
\left|y^{*}(x, t)\right| \leqslant k^{*} \quad \text { in } \Omega \times(0, T)
$$

and let us set

$$
M\left(k^{*}\right)=\max _{|a| \leqslant k^{*}}|f(a)| .
$$

We will first consider the case in which $p_{0} \in C^{0, \beta}(\bar{\Omega})$ for some $\beta \in(0,1)$ and $f$ is $C^{1}$ in $\left[-k^{*}, k^{*}\right]$.

Let us set

$$
\widetilde{g}(a, s)= \begin{cases}\frac{f(a+s)-f(a)}{f^{\prime}(a)} & \text { for }|a| \leqslant k^{*}, s \neq 0, \\ f^{s} & \text { for }|a| \leqslant k^{*}, s=0 .\end{cases}
$$

Then $\widetilde{g}$ is continuous. We claim that, for each $\eta>0$, there exists $C_{\eta}>0$ (which only depends of $\eta, k^{*}$ and the function $f$ ) such that

$$
\left\{\begin{array}{l}
\left|\widetilde{g}\left(y^{*}(x, t), s\right)\right|^{2 / 3} \leqslant C_{\eta}+\eta \log (1+|s|), \\
\forall s \in \mathbb{R}, \quad \forall(x, t) \in Q
\end{array}\right.
$$

Indeed, it will be sufficient to prove that, for each $\eta>0$, one has

$$
\left|\widetilde{g}\left(y^{*}(x, t), s\right)\right| \leqslant C_{\eta}+\eta \log ^{3 / 2}\left(1+k^{*}+|s|\right)
$$

for all $s \in \mathbb{R}$ and $(x, t) \in Q$. Let $\eta$ be given and let $s(\eta)$ be such that $s(\eta) \geqslant k^{*}+1$ and

$$
\left|\frac{f(s)}{s}\right| \leqslant \frac{2 \eta}{3} \log ^{3 / 2}(1+|s|) \quad \text { for }|s| \geqslant s(\eta) .
$$

For $|s| \leqslant 2 s(\eta)$, we have

$$
\left|\widetilde{g}\left(y^{*}(x, t), s\right)\right| \leqslant L\left(k^{*}+2 s(\eta)\right) \quad \forall(x, t) \in Q,
$$

where $L\left(k^{*}+2 s(\eta)\right)$ is a Lipschitz constant for $f$ in $\left[-k^{*}-2 s(\eta), k^{*}+\right.$ $2 s(\eta)]$. On the other hand, for $|s|>2 s(\eta)$, we see that 
E. FERNÁNDEZ-CARA, E. ZUAZUA / Ann. Inst. Henri Poincaré 17 (2000) 583-616 605

$$
\begin{aligned}
\left|\widetilde{g}\left(y^{*}(x, t), s\right)\right| & \leqslant\left|\frac{f\left(y^{*}(x, t)+s\right)}{s}\right|+\frac{1}{2 s(\eta)}\left|f\left(y^{*}(x, t)\right)\right| \\
& \leqslant \max _{\frac{|s|}{2} \leqslant|\sigma| \leqslant|s|+k^{*}}\left|\frac{f(\sigma)}{s}\right|+\frac{1}{2 s(\eta)} \max _{|a| \leqslant k^{*}}|f(a)| \\
& \leqslant \frac{3}{2} \max _{\frac{|s|}{2} \leqslant|\sigma| \leqslant|s|+k^{*}}\left|\frac{f(\sigma)}{\sigma}\right|+\frac{1}{2 k^{*}} M\left(k^{*}\right) .
\end{aligned}
$$

Here, we have used that $s(\eta)>k^{*}$ and, consequently, $|\sigma| \leqslant|s|+k^{*}$ and $|s|>2 s(\eta)$ imply $|\sigma|<3|s| / 2$. We deduce that, for $|s|>2 s(\eta)$,

$$
\left|\widetilde{g}\left(y^{*}(x, t), s\right)\right| \eta \log ^{3 / 2}\left(1+k^{*}+|s|\right)+\frac{1}{2 k^{*}} M\left(k^{*}\right) \quad \forall(x, t) \in Q .
$$

Combining (3.27) and (3.28), it is not difficult to deduce (3.24), with $C_{\eta}$ only depending of $\eta, k^{*}$ and $f$.

Notice that two different functions $f$ for which the restrictions to $\mathbb{R} \backslash\left(-k^{*}-1, k^{*}+1\right)$ and the quantities $M\left(k^{*}\right)$ and $L\left(k^{*}+1\right)$ (a Lipschitz constant in $\left.\left[-k^{*}-1, k^{*}+1\right]\right)$ coincide lead to the same constants $C_{\eta}$ in (3.25) and (3.24). This will be used below.

Let us set $Z=L^{\infty}(Q)$. Let $R>0$ be a constant whose value will be determined below. We will use the truncation function $T_{R}$, which is given as follows:

$$
T_{R}(s)= \begin{cases}s & \text { if }|s| \leqslant R, \\ R \operatorname{sgn}(s) & \text { otherwise. }\end{cases}
$$

For each $z \in Z$, we will consider the linear system

$$
\begin{cases}p_{t}-\Delta p+\widetilde{g}\left(y^{*}, T_{R}(z)\right) p=v 1_{\omega} & \text { in } Q \\ p=0 & \text { on } \Sigma \\ p(x, 0)=p_{0}(x) & \text { in } \Omega .\end{cases}
$$

Obviously, (3.29) is of the form (3.13), with $a=\widetilde{g}\left(y^{*}, T_{R}(z)\right) \in L^{\infty}(Q)$. Let us set

$$
T_{z}^{*}=\min \left[T,\left\|\widetilde{g}\left(y^{*}, T_{R}(z)\right)\right\|_{\infty}^{-2 / 3},\left\|\widetilde{g}\left(y^{*}, T_{R}(z)\right)\right\|_{\infty}^{-1 / 3}\right] .
$$

According to Theorem 3.1, there exist controls $\nu_{z} \in L^{\infty}\left(\omega \times\left(0, T_{z}^{*}\right)\right)$ such that the solution of (3.29) in $\Omega \times\left(0, T_{z}^{*}\right)$ with $v=v_{z}$ satisfies

$$
p\left(x, T_{z}^{*}\right)=0 \quad \text { in } \Omega
$$


and

$$
\left\|v_{z}\right\|_{L^{\infty}\left(\omega \times\left(0, T_{z}^{*}\right)\right)} \leqslant C_{0}\left(\Omega, \omega, T_{z}^{*},\left\|\widetilde{g}\left(y^{*}, T_{R}(z)\right)\right\|_{\infty}\right)\left\|p_{0}\right\|_{L^{2}(\Omega)},
$$

where

$$
\begin{aligned}
& C_{0}\left(\Omega, \omega, T_{z}^{*},\left\|\widetilde{g}\left(y^{*}, T_{R}(z)\right)\right\|_{\infty}\right) \\
& =\exp \left(C \left(1+\frac{1}{T_{z}^{*}}+T_{z}^{*}+\left(\left(T_{z}^{*}\right)^{1 / 2}+T_{z}^{*}\right)\left\|\widetilde{g}\left(y^{*}, T_{R}(z)\right)\right\|_{\infty}\right.\right. \\
& \left.\left.\quad+\left\|\widetilde{g}\left(y^{*}, T_{R}(z)\right)\right\|_{\infty}^{2 / 3}\right)\right)
\end{aligned}
$$

Let $A(z) \subset L^{\infty}(\omega \times(0, T))$ be the family formed by the extensions by zero to the whole interval $[0, T]$ of all these controls. Assume that $v_{z} \in A(z)$. From the definition (3.30) of $T_{z}^{*}$ and (3.31)-(3.32), it is clear that

$$
\left\|v_{z}\right\|_{L^{\infty}(\omega \times(0, T))} \leqslant \exp \left[C\left(1+\left\|\widetilde{g}\left(y^{*}, T_{R}(z)\right)\right\|_{\infty}^{2 / 3}\right)\right]\left\|p_{0}\right\|_{L^{2}(\Omega)},
$$

where $C=C(\Omega, \omega, T)$.

Now, let $\Lambda(z) \subset L^{\infty}(Q)$ be the family of the solutions of (3.29) corresponding to the controls $v_{z} \in A(z)$. Obviously, if $p_{z} \in \Lambda(z)$, one has

$$
p_{z} \equiv 0 \quad \text { in } \Omega \times\left(T_{z}^{*}, T\right) \text {. }
$$

In particular,

$$
p_{z}(x, T)=0 \quad \text { in } \Omega .
$$

In this way, we have been able to introduce a set-valued mapping $z \mapsto \Lambda(z)$. We will now check that, for some $R$, this mapping possesses at least one fixed point $p$ such that

$$
\|p\|_{\infty} \leqslant R
$$

Of course, this will imply the existence of a control $v \in L^{\infty}(\omega \times(0, T))$ such that the solution of (3.20) satisfies (3.21).

To this end, we will first see that Kakutani's Fixed Point Theorem can be applied to $\Lambda$. Indeed, it is not difficult to see that $\Lambda(z)$ is, for each $z \in L^{\infty}(Q)$, a nonempty closed convex set. From parabolic regularity, we also see that there exists a fixed compact subset $K \subset L^{\infty}(Q)$ such that

$$
\Lambda(z) \subset K \quad \forall z \in L^{\infty}(Q)
$$


(we are using here the hypothesis $p_{0} \in C^{0, \beta}(\bar{\Omega})$ ). Furthermore, $z \mapsto \Lambda(z)$ is upper hemicontinuous, i.e., the real-valued function

$$
z \mapsto \sup _{p \in \Lambda(z)}\langle\mu, p\rangle
$$

is upper semicontinuous for each bounded linear form $\mu$ on $L^{\infty}(Q)$. Consequently, Kakutani's Theorem will imply the existence of a fixed point of $\Lambda$ if we prove that, whenever $R$ is sufficiently large, any fixed point of $\Lambda$ must satisfy (3.35). We will use (1.10) (and (3.24); notice that, up to now, this has been ignored).

Thus, let $p$ be a fixed point, associated to the control $v_{p} \in A(p)$. From classical $L^{\infty}$ estimates on the solutions of (3.29), we have

$$
\begin{aligned}
\|p\|_{\infty} \leqslant & \mathrm{e}^{T_{p}^{*}\left\|\widetilde{g}\left(y^{*}, T_{R}(p)\right)\right\|_{\infty}}\left\|p_{0}\right\|_{L^{\infty}(\Omega)} \\
& +T_{p}^{*} \mathrm{e}^{T_{p}^{*}\left\|\widetilde{g}\left(y^{*}, T_{R}(p)\right)\right\|_{\infty}}\left\|v_{p}\right\|_{L^{\infty}(\omega \times(0, T))} .
\end{aligned}
$$

Consequently, taking again into account the definition of $T_{p}^{*}$ and using (3.24) and (3.33), we deduce that

$$
\begin{aligned}
\|p\|_{\infty} & \leqslant \mathrm{e}^{C\left(1+\left\|\widetilde{g}\left(y^{*}, T_{R}(p)\right)\right\|_{\infty}^{2 / 3}\right)}\left(\left\|p_{0}\right\|_{\infty}+\left\|v_{p}\right\|_{L^{\infty}(\omega \times(0, T))}\right) \\
& \leqslant \mathrm{e}^{C\left(1+C_{\eta}+\eta \log \left(1+\left\|T_{R}(p)\right\|_{\infty}\right)\right)}\left\|p_{0}\right\|_{\infty} \\
& =\mathrm{e}^{C\left(1+C_{\eta}\right)}\left\|p_{0}\right\|_{\infty}\left(1+\left\|T_{R}(p)\right\|_{\infty}\right)^{\eta C} \\
& \leqslant \mathrm{e}^{C\left(1+C_{\eta}\right)}\left\|p_{0}\right\|_{\infty}(1+R)^{\eta C}
\end{aligned}
$$

where $C=C(\Omega, \omega, T)$. Taking $\eta>0$ small enough to have $\eta C<1$, we deduce the existence of $R>0$ such that $\|p\|_{\infty} \leqslant R$. As mentioned above, this proves that, for some $v \in L^{\infty}(\omega \times(0, T))$, the corresponding solution to (3.20) satisfies (3.21). Hence, our assertion is proved when $y_{0} \in C^{0, \beta}(\bar{\Omega})$ and $f$ is $C^{1}$ in $\left[-k^{*}, k^{*}\right]$.

Notice that, in the previous argument, $R$ can be chosen depending only of $\Omega, \omega, T$, the restriction of $f$ to $\mathbb{R} \backslash\left(-k^{*}, k^{*}\right), M\left(k^{*}\right), L\left(k^{*}+1\right)$ and $\left\|p_{0}\right\|_{L^{\infty}(\Omega)}$. Thus, we have solved the controllability problem (3.20)(3.21) with $p$ and $v$ respectively bounded in $L^{\infty}(Q)$ and $L^{\infty}(\omega \times(0, T))$ by constants which only depend of these data.

Now, let us assume that $f$ is only locally Lipschitz-continuous and $p_{0} \in C^{0}(\bar{\Omega})$. We can put

$$
f=\lim _{n \rightarrow \infty} f_{n} \quad \text { uniformly in } \mathbb{R},
$$


for some locally Lipschitz-continuous functions $f_{n}$ which are $C^{1}$ in $\left[-k^{*}, k^{*}\right]$, coincide with $f$ outside $\left(-k^{*}-1, k^{*}+1\right)$ and have the same Lipschitz constants in $\left[-k^{*}-1, k^{*}+1\right]$. We can also put

$$
p_{0}=\lim _{n \rightarrow \infty} p_{0}^{n} \quad \text { uniformly in } \bar{\Omega},
$$

for some functions $p_{0}^{n} \in C^{0, \beta}(\bar{\Omega})$.

For each $n \geqslant 1$, we can argue as before and find a control $v^{n} \in$ $L^{\infty}(\omega \times(0, T))$ such that the solution $p^{n}$ to

$$
\begin{cases}p_{t}^{n}-\Delta p^{n}+f_{n}\left(y^{*}+p^{n}\right)-f_{n}\left(y^{*}\right)=v^{n} 1_{\omega} & \text { in } Q, \\ p^{n}=0 & \text { on } \Sigma, \\ p^{n}(x, 0)=p_{0}^{n}(x) & \text { in } \Omega,\end{cases}
$$

satisfies

$$
p^{n}(x, T)=0 \quad \text { in } \Omega .
$$

From the estimates we have found, we deduce that it can be assumed that

$$
\left\|v^{n}\right\|_{L^{\infty}(\omega \times(0, T))} \quad \text { and } \quad\left\|p^{n}\right\|_{\infty}
$$

are uniformly bounded. Accordingly, we can take limits in (3.36) (at least on a subsequence). In other words, it can be assumed that $p^{n}$ converges (at least) strongly in $L^{2}(Q)$ and a.e. and $v^{n}$ converges weakly-* in $L^{\infty}(\omega \times(0, T))$. This provides a control $v \in L^{\infty}(\omega \times(0, T))$ such that the corresponding solution to (3.20) satisfies (3.21). This proves our assertion when $p_{0} \in L^{\infty}(\Omega)$.

Finally, let us consider the general case, where $p_{0} \in L^{2}(\Omega)$ and $f$ is only locally Lipschitz-continuous. Let $\delta>0$ be sufficiently small and let us set $v \equiv 0$ for $t \in(0, \delta)$. Then, by parabolic regularity, the corresponding (local) solution to (3.20) satisfies $p(\cdot, \delta) \in C^{0}(\bar{\Omega})$. Now, we can apply the arguments above to $p(\cdot, \delta)$ in the interval $[\delta, T]$. Of course, this provides a control $v \in L^{\infty}(\omega \times(\delta, T))$ such that (3.21) holds. This completes the proof of Theorem 1.2.

Remark 3.2. - The arguments used for the proof of Theorem 1.2 also lead to new proofs of some known facts on the local and large time null controllability of (1.1). For instance, it can be shown that, if $f$ satisfies (1.2) and (1.3), for each $T>0$ we can find $\rho(T)>0$ such that, whenever

$$
\left\|y_{0}\right\|_{L^{2}(\Omega)} \leqslant \rho(T),
$$


we have (1.7) for some $v$. On the other hand, assuming that $f$ satisfies a good-sign condition and $f(0)=0$, we can prove zero controllability for large $T$. Indeed, due to the nonlinear absorption effect, solutions without control enter a small ball of $L^{2}(\Omega)$ at a uniform time. Then, making use of local null controllability, solutions are driven to zero (see $[8,10]$ and [15] for more details concerning these and other related questions).

\section{PROOF OF THE LACK OF APPROXIMATE CONTROLLABILITY}

This section is devoted to prove Theorem 1.3. The proof is similar to the one of Theorem 1.1. This time, it will be seen that, for an appropriate function satisfying (1.8) with $p>2$, once the initial datum $y_{0}$ and a compact set $F \subset \Omega \backslash \bar{\omega}$ are fixed, the restrictions to $F$ of the reachable states are uniformly bounded in $L^{1}(F)$. We proceed as in [14].

Let us introduce the function $f$, with

$$
f(s)=\int_{0}^{s} \log ^{p}(1+\sigma) \mathrm{d} \sigma \quad \forall s \in \mathbb{R}
$$

and $p>2$. Let us also introduce a nonnegative function $\rho \in \mathcal{D}(\Omega)$ satisfying (2.2).

Let $y_{0} \in L^{2}(\Omega)$ and $v \in L^{\infty}(\omega \times(0, T))$ be given and let $y$ be the solution to (1.1). Let us multiply by $\rho \operatorname{sgn}(y)$ the equation satisfied by $y$ and let us integrate over $\Omega$. Then

$$
\frac{\mathrm{d}}{\mathrm{d} t} \int_{\Omega} \rho|y| \mathrm{d} x=\int_{\Omega} \rho \operatorname{sgn}(y)(\Delta y) \mathrm{d} x-\int_{\Omega} \rho|f(y)| \mathrm{d} x .
$$

From Kato's inequality, we know that

$$
\int_{\Omega} \rho \operatorname{sgn}(y)(\Delta y) \mathrm{d} x \leqslant \int_{\Omega}(\Delta \rho)|y| \mathrm{d} x .
$$

We also have

$$
\left|\int_{\Omega}(\Delta \rho)\right| y|\mathrm{~d} x| \leqslant \frac{1}{2} \int_{\Omega} \rho f(|y|) \mathrm{d} x+\frac{1}{2} \int_{\Omega} \rho \widetilde{f}(2|\Delta \rho| / \rho) \mathrm{d} x,
$$


where $\widetilde{f}$ is the conjugate of the convex function $s \mapsto f(|s|)$. Arguing as in the proof of Theorem 1.1, we see that, by choosing $\rho$ appropriately (depending of $\Omega$ and $\omega$ ), the last integral in the right-hand side of (4.1) is finite (because $p>2$ ). Consequently,

$$
\frac{\mathrm{d}}{\mathrm{d} t} \int_{\Omega} \rho|y| \mathrm{d} x+\frac{1}{2} \int_{\Omega} \rho f(|y|) \mathrm{d} x \leqslant C,
$$

where $C=C(\Omega, \omega, p)$. In view of the convexity of the function $s \mapsto$ $f(|s|)$, this gives

$$
\frac{\mathrm{d}}{\mathrm{d} t} \int_{\Omega} \rho|y| \mathrm{d} x+\frac{1}{2} f\left(\int_{\Omega} \rho|y| \mathrm{d} x\right) \leqslant C .
$$

In other words, setting $z(t)=\int_{\Omega} \rho(x)|y(x, t)| \mathrm{d} x$ for all $t$, we see that

$$
z^{\prime}(t)+\frac{1}{2} f(z(t)) \leqslant C
$$

This implies that

$$
z(T)=\int_{\Omega} \rho(x)|y(x, T)| \mathrm{d} x
$$

is bounded independently of the control $v$.

Therefore, once $y_{0} \in L^{2}(\Omega)$ and an arbitrary compact set $F \subset \Omega \backslash \bar{\omega}$ are fixed, the restrictions to $F$ of the reachable states are indeed uniformly bounded in $L^{1}(F)$. This is obviously in contradiction with the density of the set of reachable states in $L^{2}(\Omega)$.

\section{PROOF OF THE APPROXIMATE CONTROLLABILITY RESULT}

The goal of this section is to prove Theorem 1.4. We will use Theorem 1.2. More precisely, we will deduce that (1.1) is approximately controllable by driving the solution exactly to the final point of an uncontrolled trajectory. In order to compensate the fact that we are in the range of nonlinearities for which blow-up may occur, we have to assume again that a globally defined solution $y^{*}$ exists. This hypothesis, 
in combination with the null controllability property, allows to keep the trajectory well defined during a large part of the time interval $[0, T]$.

First of all, notice that

$$
|f(s)| \leqslant C\left(1+|s| \log ^{3 / 2}(1+|s|)\right) \quad \forall s \in \mathbb{R},
$$

as a consequence of (1.10). Let us fix $T>0, y_{0} \in L^{2}(\Omega), y_{1} \in C^{1}(\bar{\Omega})$ (for instance) and $\varepsilon>0$ and let us try to find $v \in L^{\infty}(\omega \times(0, T))$ such that the solution of (1.1) satisfies (1.13). Obviously, it will be sufficient to consider final data $y_{1} \in C^{1}(\bar{\Omega})$, since this space is dense in $L^{2}(\Omega)$.

For any small $\delta>0$, we will consider the following auxiliary system:

$$
\begin{cases}w_{t}-\Delta w+f(w)=0 & \text { in } \Omega \times(T-\delta, T), \\ w=0 & \text { on } \partial \Omega \times(T-\delta, T), \\ w(x, T-\delta)=y_{1}(x) & \text { in } \Omega .\end{cases}
$$

There exists exactly one solution $w$ to (5.2) which is defined in the whole interval $[T-\delta, T]$. Furthermore, $w$ is bounded and there exists $\delta>0$ (small enough) such that

$$
\left\|w(\cdot, T)-y_{1}\right\|_{L^{2}(\Omega)} \leqslant \varepsilon
$$

$\delta$ depends of $\Omega,\left\|y_{1}\right\|_{L^{\infty}(\Omega)}, \varepsilon$ and the constant $C$ in (5.1). We fix $\delta$ and $w$ from now on such that (5.3) is satisfied.

Our strategy is as follows:

- For $t \in(0, T-\delta)$, we set $v=v_{1}$, where $v_{1} \in L^{\infty}(\omega \times(0, T-\delta))$ is such that $(1.1)$ is driven to $y^{*}(\cdot, T-\delta)$ at time $t=T-\delta$. In view of Theorem 1.2, such a control $v_{1}$ exists. This defines $y$ in $\Omega \times(0, T-\delta)$, with

$$
y(x, T-\delta)=y^{*}(x, T-\delta) \quad \text { in } \Omega .
$$

- We see from (5.3) that, in $[T-\delta, T]$, the problem is reduced to drive our system from $y^{*}(T-\delta)$ to the state $w(\cdot, T)$ exactly. Again in view of Theorem 1.2, there exists $v_{2} \in L^{\infty}(\omega \times(T-\delta, T))$ such that the solution to

$$
\begin{cases}y_{t}-\Delta y+f(y)=v_{2} 1_{\omega} & \text { in } \Omega \times(T-\delta, T), \\ y=0 & \text { on } \partial \Omega \times(T-\delta, T), \\ y(x, T-\delta)=y^{*}(x, T-\delta) & \text { in } \Omega\end{cases}
$$


satisfies

$$
y(x, T)=w(x, T) \quad \text { in } \Omega .
$$

Now, we set $v=v_{2}$ for $t \in(T-\delta, T)$.

Obviously, $v$ has the desired property. This completes the proof of Theorem 1.4.

\section{FURTHER COMMENTS, RESULTS AND OPEN PROBLEMS}

\subsection{On the assumption of existence of a globally defined trajectory}

The positive results of this paper, both in what concerns null and approximate controllability, require the existence of a globally defined and bounded solution for suitable $y_{0}$ and $v$. Obviously, this assumption is necessary since, otherwise, no global solution exists and the controllability problems do not make sense since they require the trace of the solution at time $t=T$ to be well defined.

The existence of global and/or stationary solutions for semilinear parabolic problems like (1.1) with $v \equiv 0$ has been the objective of intensive research. See for instance $[3,5,6,11,12]$ and the references therein. However, the wider issue of whether global solutions exist for suitable right-hand sides $v$ localized in a given subdomain $\omega$ seems to be much more open.

Of course, it would be interesting to have at our disposal sharp sufficient conditions guaranteeing the existence of globally defined solutions of (1.1) with possibly $v \neq 0$.

\subsection{On the optimality of the null controllability results}

Let us first discuss the optimality of the negative result in Theorem 1.2. Its proof is based on a localization in space of classical estimates for semilinear heat equations. Therefore, the critical growth conditions are determined by the interaction between the elliptic operator $-\Delta$ and the nonlinearity $f$. For second order O.D.E.'s, the critical growth condition is

$$
f(s) \sim|s| \log ^{2}|s| .
$$

Below this critical range, blow-up and localization phenomena do not occur. On the contrary, this can happen when the nonlinear terms grow faster at infinity. This explains the need of (1.8) with $p>2$ to prove Theorem 1.1. 
It is clear that the techniques we have used will not serve to prove negative results for nonlinearities with slower growth rate.

In what concerns the blow-up of solutions of the uncontrolled system (1.1) with $v \equiv 0$, it is well known that, when $f(s) \sim|s| \log ^{p}|s|$ with $p>2$, the blow-up is generically of pointwise nature. However, when $p<2$, the blow-up occurs globally in the whole domain $\Omega$. The case $p=2$ makes the transition: the blow-up is "regional", i.e., it occurs in an open subset of $\Omega$ (see [11] and [12]). Obviously, the arguments we have used in the proof of Theorem 1.1, that rely on the localization of energy, are compatible with pointwise blow-up but not with regional or global blow-up. Therefore, it is not reasonable to expect the same proof to extend to the case $p \leqslant 2$.

A more careful analysis of the critical nonlinearity shows that, in the class of nonlinearities that grow at infinity like

$$
f(s)=|s| \log ^{2}(1+|s|) g(s),
$$

the critical growth condition for $g$ is

$$
g(s) \sim \log ^{2}\left(\log ^{2}(1+|s|)\right)
$$

Very likely, the negative result of Theorem 1.1 still holds for nonlinearities behaving at infinity like

$$
f(s) \sim|s| \log ^{2}(1+|s|) \log ^{2}\left(\log ^{p}(1+|s|)\right)
$$

with $p>2$. But this remains to be done and, anyway, $|s| \log ^{2}(1+|s|)$ will always be a lower bound on the growth at infinity of the nonlinearities for which Theorem 1.1 might apply.

We refer to [4] for a sharp analysis of nonlinear terms behaving at infinity like iterated logarithms in the context of the exact controllability of the one-dimensional semilinear wave equation.

Let us now discuss the optimality of the positive result in Theorem 1.2. The need of the growth condition (1.10) is clear when analyzing the proof of Theorem 1.2. It is a consequence of the presence of the term

$$
\exp \left(C\|a\|_{\infty}^{2 / 3}\right)
$$

in the estimate of the cost of null controllability of linear heat equations.

Roughly speaking, if the estimate of the cost of controllability contains a factor of the form $h\left(\|a\|_{\infty}\right)$, the growth of nonlinearities of order 
$s h^{-1}(s)$ is critical. This explains the need of (1.10). Note that the estimate of the cost of null controllability for the linear heat equation with a potential also contains a factor of the order of

$$
\exp \left(C\left(T^{1 / 2}+T\right)\|a\|_{\infty}\right) .
$$

According to this, the critical growth for $f$ would be $f(s) \sim s \log (1+$ $|s|)$. However, we have been able to go beyond this level and reach the growth conditions (1.10) by choosing a sufficiently small effective time of control.

The presence of the factor (6.1) in the estimate of the cost of null controllability is a consequence of the obervability estimate (3.2). It arises naturally when applying Carleman inequalities. Whether or not this factor may be dropped (or relaxed) in the observability inequalities in order to improve the growth condition (1.10) is an interesting open problem.

\subsection{On the optimality of the approximate controllability result}

In what concerns the optimality of the negative result in Theorem 1.3, the same remarks of Section 6.2 above apply.

In what concerns the growth condition (1.4) in Corollary 1.1, it is important to recall that it excludes nonlinearities leading to blow-up phenomena (see [5]). In Theorem 1.4, we have relaxed this growth condition, but at the prize of imposing the additional condition that a globally defined and bounded solution exists.

\subsection{Finite-approximate controllability}

The techniques of this paper also serve to analyze the finite-approximate controllability problems for semilinear parabolic systems.

Assume a finite-dimensional space $E \subset L^{2}(\Omega)$ is given. Let us denote by $\pi_{E}$ the orthogonal projector on $E$. It will be said that (1.1) is finiteapproximate controllable at time $T$ (with respect to $E$ ) if, for any $y_{0} \in$ $L^{2}(\Omega), y_{1} \in L^{2}(\Omega)$ and $\varepsilon>0$, there exists a control $v \in L^{\infty}(\omega \times(0, T))$ such that the solution of system (1.1) is globally defined in $[0, T]$ and satisfies

$$
\pi_{E}(y(T))=\pi_{E}\left(y_{1}\right), \quad\left\|y(T)-y_{1}\right\|_{L^{2}(\Omega)} \leqslant \varepsilon .
$$

Obviously this a stronger notion than the approximate controllability property. The cost of finite-approximate controllability in $L^{2}(\omega \times(0, T))$ 
for linear systems like (3.13) was addressed in [9]. Assuming that $E$ is a finite-dimensional subspace of $H_{0}^{1}(\Omega)$ and (for instance) $y_{0}=0$ and $y_{1} \in H^{2}(\Omega) \cap H_{0}^{1}(\Omega)$, the finite-approximate controllability property was proved with the following estimate on the control:

$$
\begin{aligned}
& \|v\|_{L^{2}(\omega \times(0, T))} \\
& \quad \leqslant \exp \left[C\left[N\left(T,\|a\|_{\infty}\right)+\left(1+\Lambda_{E}\right) \frac{M\left(\|a\|_{\infty}, y_{1}\right)}{\varepsilon}\right]+\Lambda_{E}\right]\left\|y_{1}\right\|_{L^{2}(\Omega)},
\end{aligned}
$$

with

$$
\begin{gathered}
N\left(T ;\|a\|_{\infty}\right)=1+\frac{1}{T}+T\|a\|_{\infty}+\|a\|_{\infty}^{2 / 3}, \\
M\left(\|a\|_{\infty}, y_{1}\right)=\|a\|_{\infty}\left\|y_{1}\right\|_{L^{2}(\Omega)}+\left\|\Delta y_{1}\right\|_{L^{2}(\Omega)}, \\
\Lambda_{E}=\left\|\pi_{E}\right\| \exp \left[T\left[\mu(E)+\|a\|_{\infty}\right] \mathrm{e}^{T\|a\|_{\infty} / 4}+C N\left(T,\|a\|_{\infty}\right)\right], \\
\mu(E)=\max _{\varphi \in E \backslash\{0\}} \frac{\|\nabla \varphi\|_{L^{2}(\Omega)}^{2}}{\|\varphi\|_{L^{2}(\Omega)}^{2}} .
\end{gathered}
$$

Observe that the constant in (6.2) is of the order of $\exp \left[C_{1} \exp \left[C_{2} \times\right.\right.$ $\left.\left.\exp \left[C_{3}\|a\|_{\infty}\right]\right]\right]$. Thus, it can be expected that the fixed point techniques we have used in this paper serve to prove finite-approximate controllability at least for functions $f$ satisfying

$$
\frac{f(s)}{|s| \log (\log (\log (1+|s|)))} \rightarrow 0 \quad \text { as }|s| \rightarrow \infty .
$$

However, this has to be done.

\subsection{Extensions}

The results we have proved may be extended to other situations including boundary controls, parabolic operators with variable smooth coefficients, initial data in $L^{p}$ with $p$ not neccesarily equal to 2 , etc.

\section{REFERENCES}

[1] Anita S., Barbu V., Internal null controllability of nonlinear heat equation, Preprint, 1999. 
[2] Barbu V., Exact controllability of the superlinear heat equation, Preprint, 1998.

[3] Brezis H., Cazenave Th., Martel Y., Ramiandrisoa A., Blow up for $u_{t}-\Delta u=g(u)$ revisited, Adv. Differential Equations 1 (1) (1996) 73-90.

[4] Cannarsa P.M., Komornik V., Loreti P., Well posedness and control of the semilinear wave equation with iterated logarithms, ESAIM: COCV (to appear).

[5] Cazenave T., Haraux A., Equations d'évolution avec nonlinéarité logarithmique, Ann. Fac. Sci. Toulouse 2 (1980) 21-51.

[6] Cazenave T., Haraux A., Barbu V., Introduction aux Problèmes d'Évolution Semilinéaires, Mathématiques \& Applications, Ellipses, Paris, 1989.

[7] Fabre C., Puel J.P., Zuazua E., Approximate controllability of the semilinear heat equation, Proc. Royal Soc. Edinburgh 125A (1995) 31-61.

[8] Fernández-Cara E., Null controllability of the semilinear heat equation, ESAIM: COCV 2 (1997) 87-107.

[9] Fernández-Cara E., Zuazua E., The cost of approximate controllability for heat equations: The linear case, Adv. Differential Equations (to appear).

[10] Fursikov A., Imanuvilov O.Yu., Controllability of Evolution Equations, Lecture Notes, Vol. 34, Seoul National University, Korea, 1996.

[11] Galaktionov V., On blow-up and degeneracy for the semilinear heat equation with source, Proc. Royal Soc. Edinburgh 115A (1990) 19-24.

[12] Galaktionov V., Vázquez J.L., Regional blow-up in a semilinear heat equation with convergence to a Hamilton-Jacobi equation, SIAM J. Math. Anal 24 (5) (1993) $1125-1276$.

[13] Glass O., Sur la contrôlabilité des fluides parfaits incompressibles, Ph.D. Thesis, Université de Paris XI, Orsay, France, January 2000.

[14] Henry J., Contrôle d'un réacteur enzymatique à l'aide de modèles à paramètres distribués: Quelques problèmes de contrôlabilité de systèmes paraboliques, Ph.D. Thesis, Université Paris VI, 1978.

[15] Imanuvilov O.Yu., Exact boundary controllability of the parabolic equation, Russian Math. Surveys 48 (1993) 211-212.

[16] Khapalov A., Some aspects of the asymptotic behavior of the solutions of the semilinear heat equation and approximate controllability, J. Math. Anal. Appl. 194 (1995) 858-882.

[17] Zuazua E., Exact boundary controllability for the semilinear wave equation, in: Brezis H., Lions J.L. (Eds.), Nonlinear Partial Differential Equations and their Applications, Vol. X, Pitman, 1991, pp. 357-391.

[18] Zuazua E., Finite dimensional null-controllability of the semilinear heat equation, J. Math. Pures et Appl. 76 (1997) 237-264.

[19] Zuazua E., Exact controllability for the semilinear wave equation in one space dimension, Ann. IHP, Analyse non Linéaire 10 (1996) 109-129. 